Instrumentation and Measurement

Technology Conference - IMTC 2007

Warsaw, Poland, May 1-3, 2007

\title{
A Constant Temperature Thermoresistive Sigma-Delta Anemometer
}

\author{
Will R. M. Almeida ${ }^{1}$, Georgina M. Freitas ${ }^{1}$, Lígia S. Palma ${ }^{3}$, Sebastian Y.C. Catunda ${ }^{2}$, Raimundo C. S. \\ Freire $^{1}$, Hassan Aboushady ${ }^{4}$, Francisco F. Santos ${ }^{1}$, Amauri Oliveira ${ }^{3}$. \\ ${ }^{1}$ Universidade Federal de Campina Grande, Unidade Acadêmica de Engenharia Elétrica, Campina Grande - PB, Brazil. \\ ${ }^{2}$ Universidade Federal do Maranhão, Departamento de Engenharia de Eletricidade, São Luis - MA, Brazil. \\ ${ }^{3}$ Universidade Federal da Bahia, Departamento de Engenharia Elétrica, Salvador - BA, Brazil. \\ ${ }^{4}$ University of Paris VI, Pierre \& Marie Curie, LIP6/ASIM Laboratory, 75252 Paris, France \\ Email: \{willalmeida, rcsfreire,ffsantos\}@dee.ufcg.edu.br, geo maciel@hotmail.com, \\ catunda@dee.ufma.br, \{ligia,amauri\}@ufba.br,Hassan.Aboushady@lip6.fr.
}

\begin{abstract}
In this paper we propose a feedback measurement system, with thermoresistive sensor, based on sigma-delta modulation. The system uses a one-bit sigma-delta modulator for which a considerable part of the conversion functions is performed by a thermoresistive sensor. The sensor is modelled using the power balance principle and the constant temperature measurement method is employed. This transducer architecture is able to realize digital measurement of physical quantities that interacts with the sensor: temperature, thermal radiation and fluid velocity. This paper presents simulations and validates the structure of the sigmadelta converter as an anemometer. The advantage to directly convert physical quantities to digital, with the sensor as part of the $A$ to $D$ converter, makes possible the implementation of this measurement system in an integrated circuit. Another advantage of the IC implementation of this structure is the possibility to reduce noise interference due to discrete component connections in a printed circuit board.
\end{abstract}

Keywords - Thermoresistive sensor, Anemometer, Sigma-delta modulation, Constant temperature architecture, Microsensors.

\section{INTRODUCTION}

Negative feedback system configurations with thermoresistive sensor using the power balance principle have been employed in the measurement of thermal radiation [1-3], fluid velocity [4,5], and temperature [6]. In the most used method, called constant temperature, the sensor is heated by Joule effect to a chosen operating temperature and the thermal radiation, fluid velocity, or temperature variation is compensated by a change in electrical heating due to the employed negative feedback, and the sensor is kept at an almost constant temperature.

The Wheatstone bridge, with the sensor in one of its arms, is the most used configuration to implement the measurement system with a sensor heated to a constant temperature, either with continuous or pulsed signal (PWM) feedback. Another attractive possibility is the use of a sigma-delta configuration, with the sensor as part of the sigma-delta feedback loop [8].

The 1-bit sigma-delta modulator is a feedback analog to digital configuration where the output signal is an oversampled digital version of the analog input signal [9]. Sigma-delta modulator has been employed, in signal processing, to convert analog signals to digital using simple analog circuitry and with relatively complex digital circuits. These sigma-delta converters are recognized to be robust, simple architecture and high performance $\mathrm{A} / \mathrm{D}$ converters.

This work presents an anemometer configuration composed of a 1-bit sigma-delta modulator where some of its constituting blocks are substituted by the thermoresistive microsensor. Simulation results are presented for two structures of sigma-delta converters using standard input signals (step and sine wave). The first simulated structure has only the functional characteristics of the physical system, which is used for studying the system behaviour, but it cannot be implemented on an integrated circuit. The second simulated structure is similar to the first one but can be implemented on an integrated circuit.

\section{PROBLEM DEFINITION}

The dynamic heat equation for a thermoresistive sensor is expressed by $[3,4]$ :

$$
P_{e}+\alpha H S=h S\left(T_{s}-T_{f}\right)+m c \frac{d T_{s}}{d t}
$$

In (1), $\alpha S H$ is the incident thermal radiation absorbed by the sensor, $P_{e}=I_{s}^{2} R_{s}$ is the electrical power delivered to the sensor, $h$ is the heat transfer coefficient referred to the sensor surface area $S, T_{s}$ is the sensor temperature, $T_{f}$ is the surrounding fluid temperature, $m$ is the sensor mass, $c$ is the sensor specific heat. The sensor temperature, $T_{s}$, can be given by:

$$
T_{s}=\frac{1}{m c} \int_{-\infty}^{t} \alpha H S+I_{s}^{2} R_{s}-h S\left(T_{s}-T_{f}\right) d t
$$

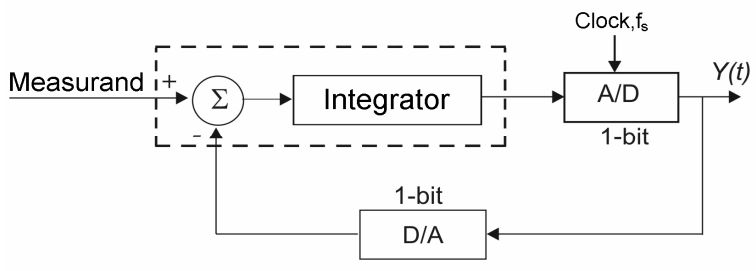

Figure 1. Block diagram of a first order sigma-delta modulator. 
Figure 1 shows the block diagram of a first-order sigmadelta modulator. The summing and integrating blocks are in evidence, and show a similarity with (2).

The idea of including the microsensor into a 1-bit firstorder sigma-delta loop comes from the mentioned similarity and from the fact that the sensor temperature response curve leads to an almost exponential function in response to a squared current step, for small amplitudes. Thus, considering that the sampling frequency, $f_{s}$, is much greater than the sensor linear transfer function pole, this exponential can be approximated by an integration function in which the gain is the exponential function initial slope [3]. Considering that the step responses for an ideal integrator and for the exponential function are almost coincident up to $10 \%$ of the exponential time constant, some studies were carried out analyzing the small signals model for the sigma-delta converters employed for the measurement of thermal radiation and environment temperature. Based on these studies and assuming that $H$ is equal to zero, we propose a structure based on sigma-delta modulation for estimating the fluid velocity, $\vartheta$. Hence, $T_{s}$, can be expressed by:

$$
T_{s}=\frac{1}{m c} \int_{-\infty}^{t} I_{s}^{2} R_{s}-h S\left(T_{s}-T_{f}\right) d t .
$$

The thermoresistor microsensor used has a positive temperature coefficient, PTC. The thermal behavior mathematical model of the PTC is given by [4]:

$$
R_{s}=R_{o}\left[1+\beta\left(T_{s}-T_{f}\right)\right]
$$

where $R_{o}$ is the sensor resistance at $0{ }^{\circ} \mathrm{C}$ and $\beta$ is the thermal coefficient, which depends on the sensor material.

The heat transfer coefficient, $h$, can be given by: [10]

$$
h=a+b \vartheta^{n} \text {, }
$$

where $a, b$ and $n$ are constants to be determined experimentally for a given microsensor specific operating conditions [11] and $\vartheta$ is a fluid velocity.[12].

Combining (3) and (5), considering the substitutions: $I^{2}{ }_{\mathrm{s}}$ for $Y_{s}$, assuming the condition of static thermal balance and

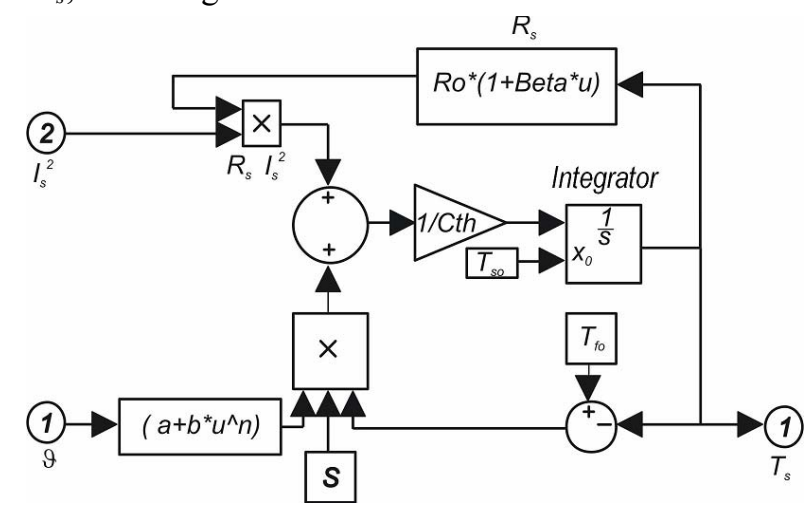

Figure 2. Block diagram of the PTC sensor model. that the sensor temperature is kept almost constant, one can write:

$$
\vartheta=\left\{\frac{1}{b}\left[\frac{Y_{s} R_{s}}{S\left(T_{s}-T_{f}\right)}-a\right]\right\}^{1 / n} .
$$

Figure 2 shows the thermoresistive microsensor behavioural model, given by (3), used as a component in the continuous current model of the proposed anemometer. From Figure 2, it can be observed that $\vartheta$ and the sensor squared current, $I_{s}^{2}$, are the input signals whereas the sensor temperature, $T_{s}$, is the output signal.

\section{A. Continuous current modulator $\Sigma-\Delta$ model}

The block diagram of the continuous current (CC) $\Sigma-\Delta$ modulator model is shown in Figure 3, for which $T_{s}(t)$ is the sensor temperature and $\vartheta(t)$ is the measurand. $T_{s o}$ and $Y_{s o}$ are the sensor temperature and the sensor squared current, respectively, in thermal equilibrium. The current gain is obtained from (5) with $\vartheta_{\text {amax }}$ and $\vartheta_{\text {amin }}$ being, respectively, the upper and lower limit of the fluid speed range. The Current gain can be expressed by:

$$
\Delta Y_{s o}=\frac{S b}{R_{s o}}\left[\left(T_{s}-T_{f}\right)\left(\frac{\vartheta_{a \max }{ }^{n}-\vartheta_{a \min }{ }^{n}}{2}\right)\right] .
$$

As shown in Figure 3, there are two inputs at proposed model that makes its implementation unpractical: a) the first one is the sensor model squared current input; b) the second one is a non-electrical quantity $\left(T_{s}\right)$ at the quantizer input. Therefore, this continuous current model works for validating the sigma-delta anemometer behavior and for the comparison with the model to be implemented.

\section{B. Pulsed current modulator $\Sigma-\Delta$ model.}

Figure 4 shows the pulsed current (PC) $\Sigma-\Delta$ modulator model, a modified version of the continuous current model

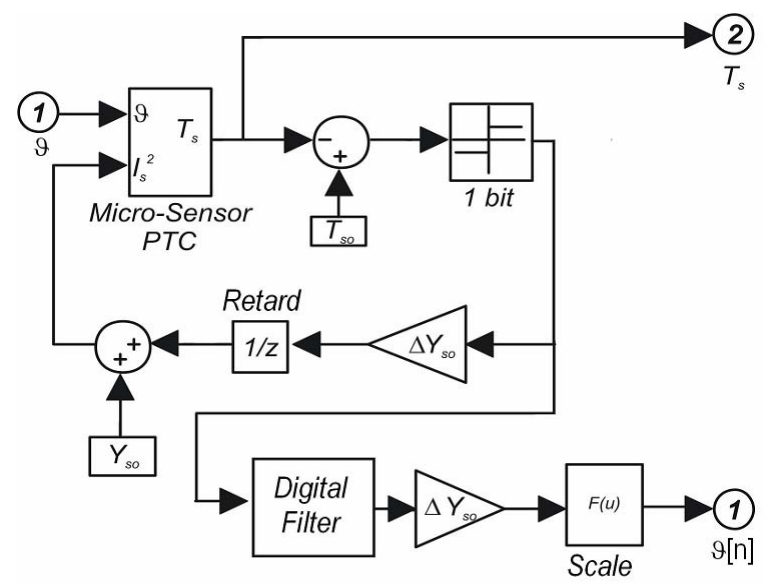

Figure 3. Block diagram of the continuous current $\Sigma-\Delta$ modulator model. 
including a pulse width modulator (PWM), and a sample and hold block for the voltage during current pulse. To obtain this model, we firstly substitute sensor squared current for a pulse width modulated current $\left(I_{\text {sef }}^{2}\right)$, which is given by:

$$
I_{\text {sef }}^{2}=I_{m}^{2} \frac{\Delta \delta}{T_{P W M}}
$$

where $I_{m}, T_{P W M}$ and $\Delta \delta$ are the pulse amplitude, period and pulse width proportional to the PWM input, respectively. Secondly, we substitute the sensor model output, $T_{s}(t)$, for the sensor voltage, $V_{s}(t)$.

In the pulsed current model, the PWM generated current substitutes the model input $I_{s}^{2}$ with a pulsed current (Figure 4), which is proportional to $\Delta \delta$ and $I_{\text {sef }}^{2}$. The PWM generates only two pulse widths, for the quantizer outputs +1 and -1 . In thermal equilibrium, the pulse width has a theoretical value equal to $50 \%$ of $T_{P W M}$. The information of current gain is now in the pulse width, which has a nonlinear relationship with the air velocity, given by:

$$
\frac{\Delta_{\delta}}{T_{P W M}}=\frac{1}{I_{m}^{2} R_{s o}}\left[S\left(T_{S}-T_{f}\right)\left(a+\frac{b\left(\vartheta_{a \max }{ }^{n}-\vartheta_{a \min }{ }^{n}\right)}{2}\right)\right]
$$

The Figure 4 shows the pulsed current $\Sigma-\Delta$ modulator model, a modified version of continuous current transducer model including a PWM, and a sample \& hold (Zero order Hold) for the voltage during PWM current pulse. The Delay block (transport delay) is included, only in simulation, to guarantee the sampling during PWM current pulse. The sampled voltage between sensor terminals, in thermal equilibrium condition, is given by.

$$
V_{s o}=I_{m} R_{s o}
$$

If $V_{s o}$ is kept constant by the feedback loop, $R_{s}$ is also constant and therefore, the sensor temperature too.

\section{SIMULATION RESULTS}

The PTC sensor parameters used to test both systems

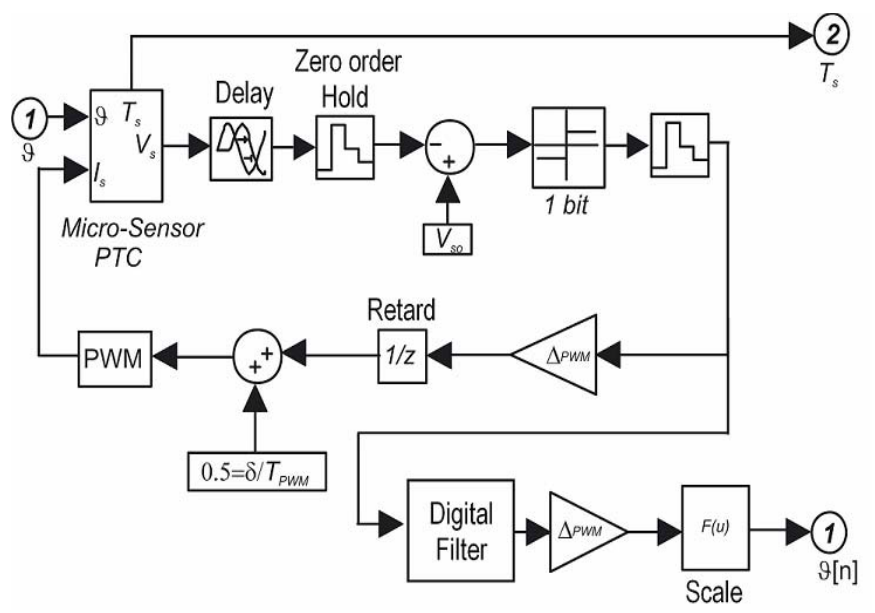

Figure 4. Block diagram of the Pulsed current $\Sigma-\Delta$ modulator model. were: $\beta=0.000784^{\circ} \mathrm{C}^{-1}, R_{\mathrm{o}}=102 \Omega, m c=292.5610^{-12} \mathrm{~J}^{\circ} \mathrm{C}^{-1}$, $\mathrm{S}=4 \times 10^{-9} \mathrm{~m}^{2}$ [11]. Also, for both system: $T_{s o}=80^{\circ} \mathrm{C}, \vartheta_{\text {amin }}=$ $0 \mathrm{~m} / \mathrm{s}, \vartheta_{\text {amax }}=20 \mathrm{~m} / \mathrm{s}, T_{f o}=30^{\circ} \mathrm{C}, p=1.17 \times 10^{6} \mathrm{rad} / \mathrm{s}$, OSR $($ Over Sampling Rate $)=256$.

The continuous current (CC) model characteristics were: $Y_{s o}=4.6110^{-4} \mathrm{~A}^{2}, \Delta Y_{s o}=2.9510^{-4} \mathrm{~A}^{2}$. The pulsed current (PC) model characteristics were: $I_{m}=0.03 \mathrm{~A}, T_{P W M}=0.0125$.

A fluid velocity step degree from $5 \mathrm{~m} / \mathrm{s}$ to $10 \mathrm{~m} / \mathrm{s}$ was applied to the input of continuous current system and to the input of the pulsed current system. The output sensor temperature, $T_{s}$, and the estimated fluid velocity, $v[\mathrm{n}]$, were observed in both systems. Figure 5 and Figure 6 show that sensor temperature quickly gets to around $80{ }^{\circ} \mathrm{C}$ and remains around this temperature value.

The detail, in Figure 7 and Figure 8, shows that this

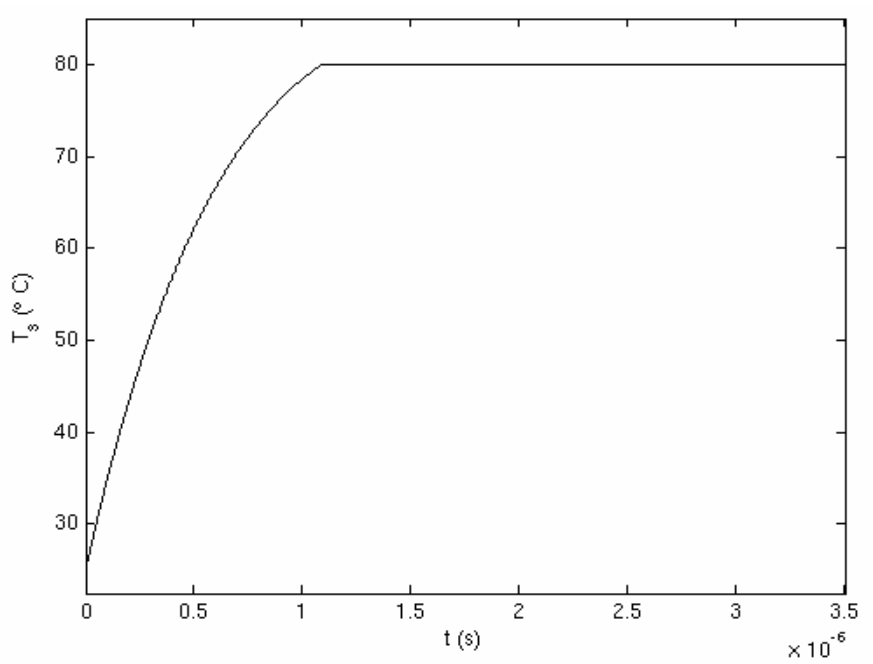

Figure 5. Continuous current model: Sensor temperature response to a step of fluid velocity.

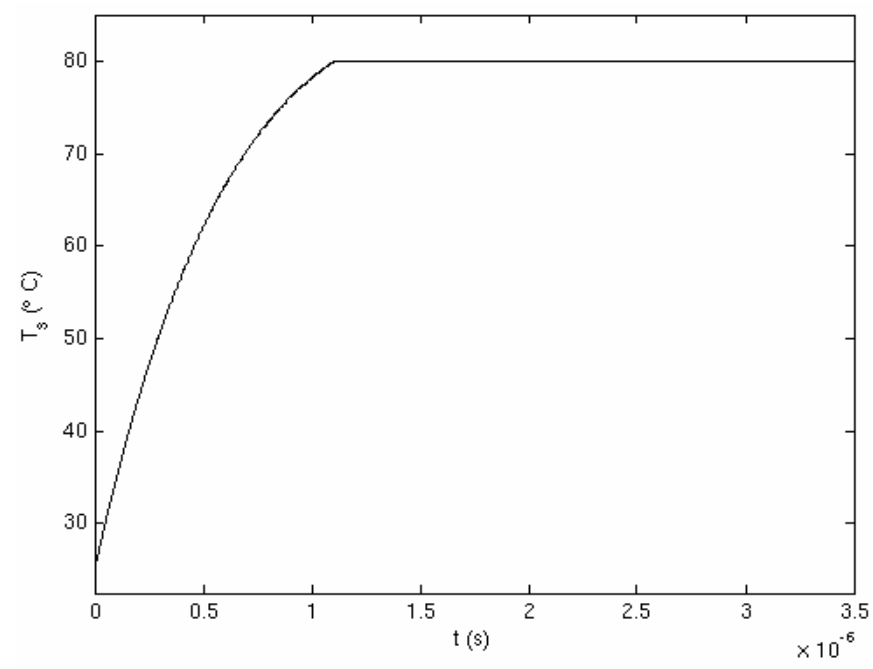

Figure 6. Pulsed current model: Sensor temperature response to a step of fluid velocity. 
variation remains between $79.991{ }^{\circ} \mathrm{C}$ and $80.004{ }^{\circ} \mathrm{C}$ for the continuous current system and between $79.986{ }^{\circ} \mathrm{C}$ and $80.009^{\circ} \mathrm{C}$ for the pulsed current system. This variation depends on input fluid velocity amplitude and system resolution. Also, it can be observed that the sensor temperature signal pattern is similar to the integrator output signal pattern of the $\Sigma-\Delta$ modulator,

Figure 9 and Figure 10 show the estimated fluid velocity for the continuous current system and for the pulsed current system, respectively. The average value of fluid velocity begins at $5 \mathrm{~m} / \mathrm{s}$ and moves to nearly $10 \mathrm{~m} / \mathrm{s}$ after the step time.

From Figure 11, the estimated fluid velocity response to a step input can be detailed observed. The value of the estimated fluid velocity for the CC model is around $10 \mathrm{~m} / \mathrm{s}$ and for the PC model, it varies between 9.98 and $10.02 \mathrm{~m} / \mathrm{s}$.

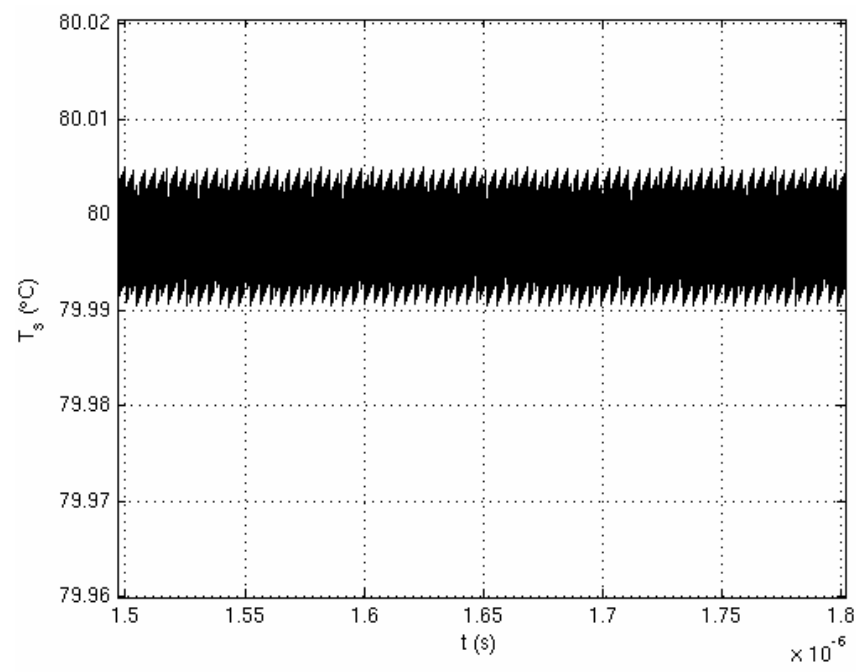

Figure 7. CC model: Sensor temperature response detail to a step of velocity.

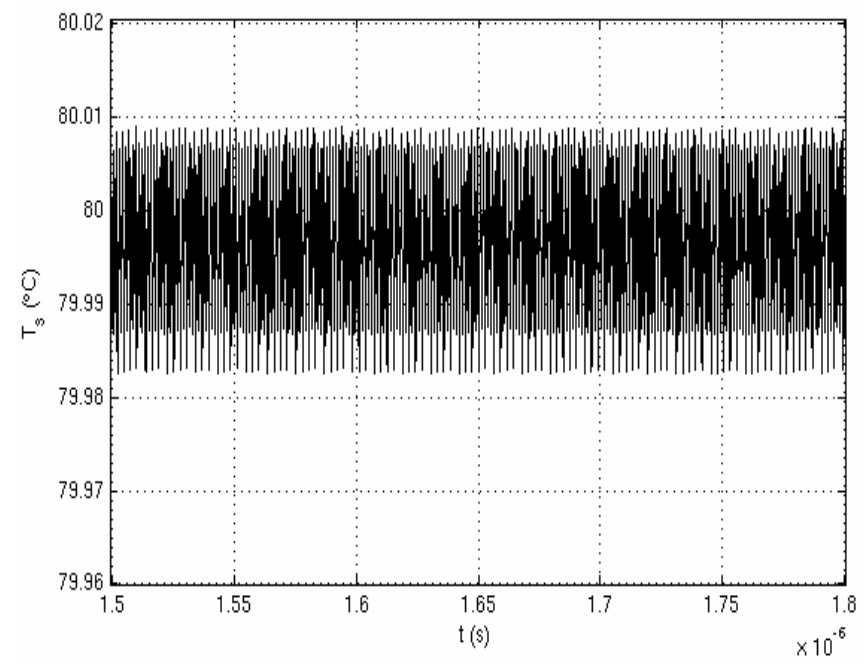

Figure 8. PC model: Sensor temperature response detail to a step of velocity.
The PC model estimation error is due to the system dynamic response to the pulsed input current. This error could be reduced applying a shorter pulse period in the PWM, although, the minimum time period is limited by the chosen technology to be employed.

A sine wave signal (representing the fluid velocity) given by $\vartheta(t)=\left[10+10 \sin \left(\pi t / 10^{5}\right)\right] \mathrm{m} / \mathrm{s}$ was applied to the continuos and pulsed current system input and the estimated fluid velocities, $\vartheta[n]$, were observed as shown in Figure 12 and Figure 13, respectively.

Figure 14 shows a detail of the estimated fluid velocity response to a sine wave input for both $\mathrm{CC}$ and $\mathrm{PC}$ models. The PC model response shows a considerable loss of dynamic resolution near to the positive upper limit of the measurement range, when compared to the $\mathrm{CC}$ response. This is due to an approximation where the temperature is

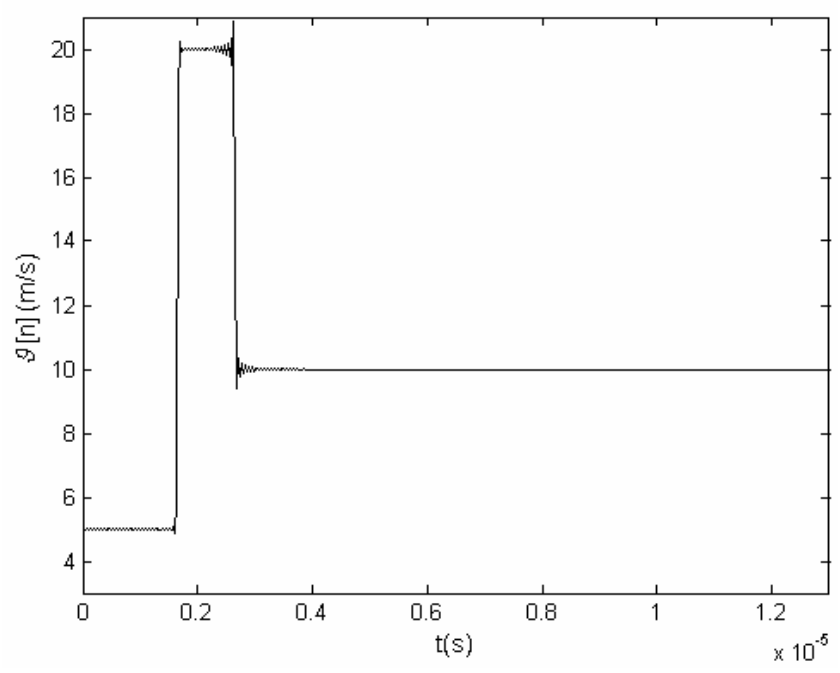

Figure 9. CC model: Estimated velocity, response to a sine wave velocity.

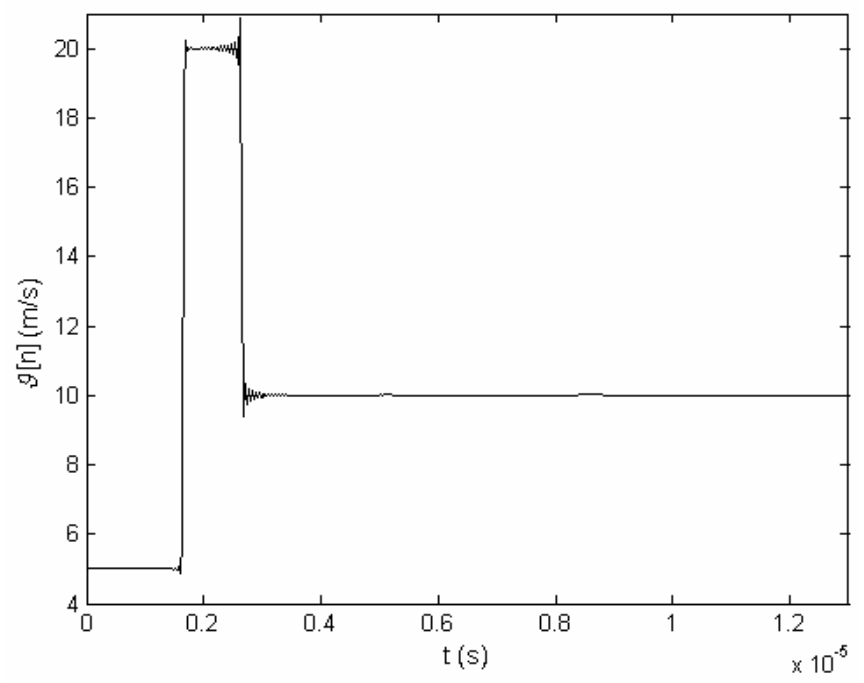

Figure 10. PC model: Estimated velocity, response to a sine wave of velocity. 
considered to be constant, in the fluid velocity estimation. In practice, the temperature is not constant but varies around a value, which is, in this case, $80^{\circ} \mathrm{C}$. Hence, it can be observed that the continuous current model presents a better resolution than the pulsed one

\section{CONCLUSION}

The pulsed current fluid velocity architecture, as an equivalent system to the continuous current architecture, realized the expected $\mathrm{A} / \mathrm{D}$ conversion with good resolution when compared to the $\mathrm{CC}$ architecture.

The simulation results obtained for the PC model were satisfactory, either for the sensor temperature variation as for the fluid speed estimation. The PC architecture does not need

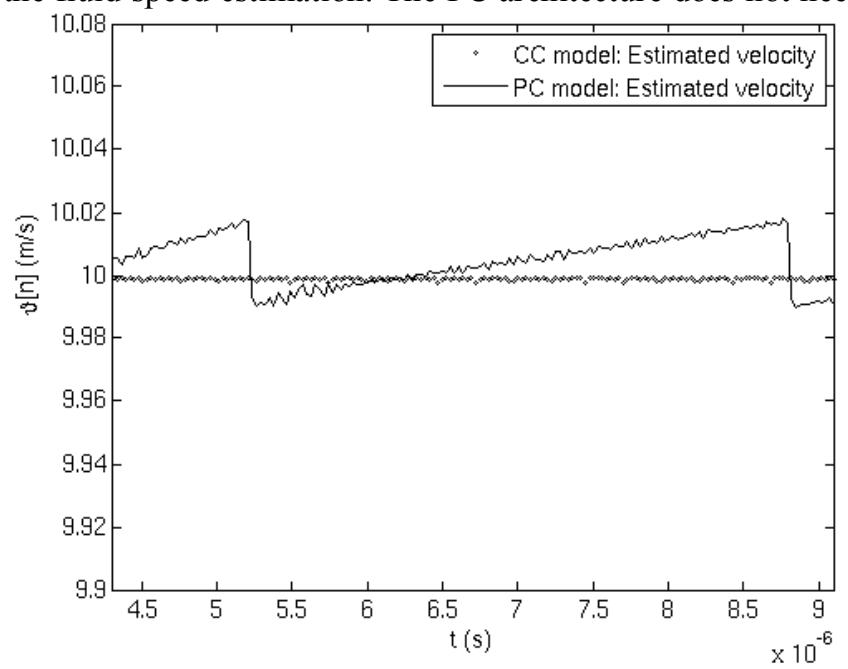

Figure 11.PC and CC systems estimated fluid velocity response to a step input, after output stabilization.

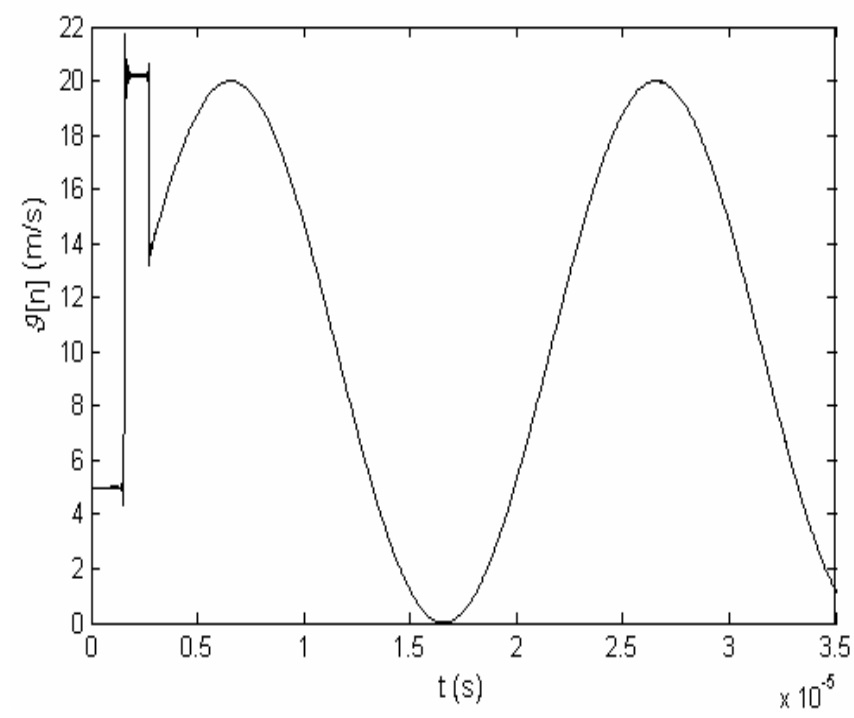

Figure 12. Estimated fluid velocity corresponding to a sinusoidal input applied to the continuos current model. to employ a 1-bit $\mathrm{D} / \mathrm{A}$ converter, since this function is carried out by the PWM block. As the CC architecture is not a feasible one, the PC architecture has shown to be acceptable for conceiving an integrated circuit anemometer, integrating both circuits and sensor in a single chip, which directly converts the physical quantity to a digital signal.

\section{ACKNOWLEDGMENT}

The authors wish to thank CAPES, CNPq, CAPES/COFECUB and FAPESQ/PRONEX for financial support and the award of fellowships during investigation period.

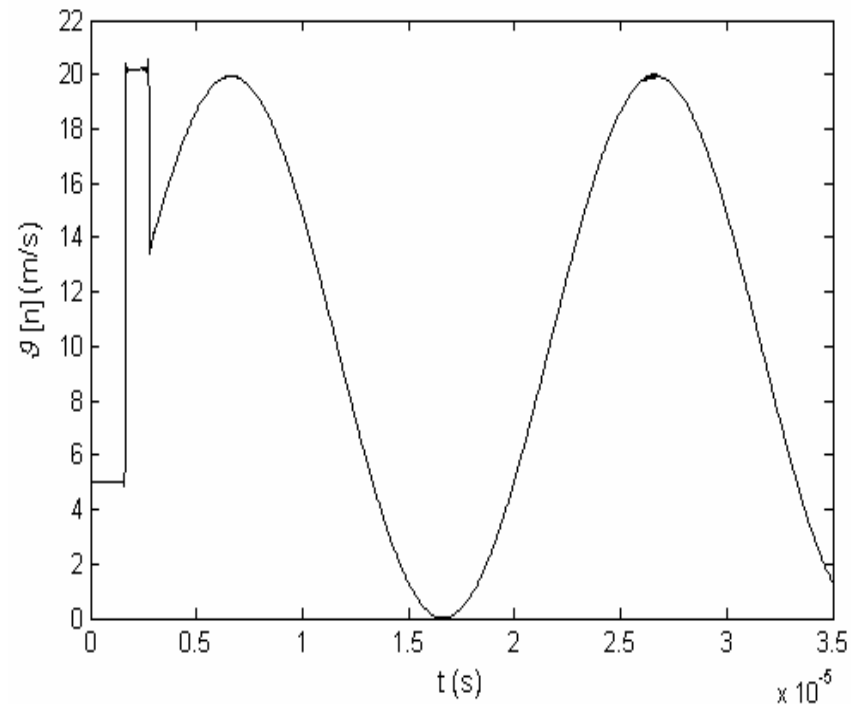

Figure 13. Estimated fluid velocity corresponding to a sinusoidal input applied to the pulsed current model.

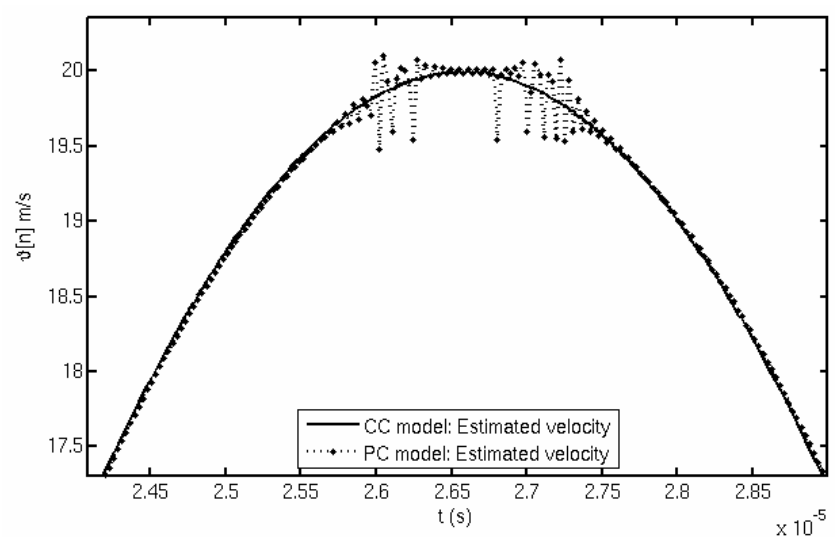

Figure 14. Detail of both system estimated fluid velocity response to a sinusoidal input. 


\section{REFERENCES}

[1] A. Oliveira,G.S. Deep, A.M.N. Lima, R.C.S. Freire, A feedback I2controlled constant temperature solar radiation meter, in: Proc. IEEEIMTC, vol. 2, 1998, pp. 1062-1066.

[2] R.C.S. Freire, G.S. Deep, C.C. Farias, Electrical equivalence solar radiometer configurations, in: Proc. XI Congresso Brasileiro de Automatica, vol. 3, 1996, pp. 1249-1254.

[3] L. S. Palma, A. Oliveira, A. S. Costa, R. C .S. Freire, A. C. Lima, A Constant Temperature Operation Thermoresistive Sigma-Delta Solar Radiometer. Proceedings 10th IMEKO TC7 International Symposium on Advances of Measurement Science. Saint Petersburg : Sergey Muravyov- Tomsk Polytechnic University, 2004. v. 1. p. 199-204.

[4] H. Fujita, T. Ohhashi, M. Asakura, M. Yamada, K. Watanabe, A thermistor anemometer for low-flow-rate measurements, IEEE Trans. Instrum. Meas. 44 (3) (1995) 779-782.

[5] R. P. C. Ferreira, R. C. S. Freire, G. S. Deep, J. S. Rocha Neto, A. Oliveira, Fluid temperature compensation in a hot wire anemometer using a single sensor, in: Proc. IEEE-IMTC,vol. 1, 2000, pp. 512-517.
[6] L. S. Palma, A. Oliveira, A.S. Costa, A.Q. Andrade Jr., C.V.R. Almeida, M.E.P.V. Zurita, R.C.S. Freire, Implementation of a feedback I2-controlled constant temperature environment temperature meter, Sensors 3 (10) (2003) 498-503.

[7] K.A.A. Makinwa, J.H. Huijsing, Constant power operation of a twodimensional flow sensor using thermal sigma-delta modulation techniques, in: Proc. IEEE-IMTC, Budapest, 2001, pp. 1577-1580.

[8] A. Oliveira, A. S. Costa, L. S. Palma, A. C. Lima, R.C.S. Freire, A Contant Temperature Operation Thermo-Resistive Sigma-Delta Transducer. In: IMTC/2004 - Instrumentation and Measurement Technology Conference, 2004, Como - Italy. Instrumentation and Measurement Technology Conference. Piscatway : IEEE, 2004. v. 1. p. 1175-1180.

[9] P.M. Aziz, H.V. Sorensen, J.V.D. Spiegel, An overview of sigma-delta converters, IEEE Signal Process. Mag. (1996)61-84.

[10] R. P. C. Ferreira, R. C. S. Freire, G. S. Deep, J. S. Rocha Neto, A. Oliveira, Hot-Wire Anemometer With Temperature Compensator Using Only One Sensor. Ieee Transactions On Instrumentation And Measurement, Estados Unidos, V. 50, N. 4, P. 954-958, 2001.

[11] A. C. O. Júnior. Desenvolvimento de Micro-Aquecedores Compatíveis com Tecnologia de Microeletrônica para Aplicação em Transdutores Térmicos. 2003. Dissertação (Mestrado em Engenharia Elétrica) Universidade Estadual de Campinas.

[12] J. O. Hinze, Turbulence, New York: Mc Graw-Hill, 1975, ch. 2. 